\section{(6) OPEN ACCESS}

\title{
Progressive cone and cone-rod dystrophies: clinical features, molecular genetics and prospects for therapy
}

\author{
Jasdeep S Gill, ${ }^{1}$ Michalis Georgiou, ${ }^{1,2}$ Angelos Kalitzeos, ${ }^{1,2}$ Anthony T Moore, ${ }^{1,3}$ \\ Michel Michaelides ${ }^{1,2}$
}

\begin{abstract}
- Additional material is published online only. To view please visit the journal online (http://dx.doi.org/10.1136/ bjophthalmol-2018-313278).

'UCL Institute of Ophthalmology, University

College London, London, UK ${ }^{2}$ Moorfields Eye Hospital NHS Foundation Trust, London, UK ${ }^{3}$ Ophthalmology Department, University of California San Francisco School of Medicine, San Francisco, California, USA
\end{abstract}

Correspondence to Professor Michel Michaelides, UCL Institute of Ophthalmology, London EC1V 9EL, UK; michel. michaelides@ucl.ac.uk

Received 24 September 2018 Revised 23 November 2018 Accepted 29 November 2018 Published Online First 24 January 2019

Check for updates

(C) Author(s) (or their employer(s)) 2019. Re-use permitted under CC BY. Published by BMJ.

To cite: Gill JS, Georgiou M, Kalitzeos A, et al.

$\mathrm{Br} J$ Ophthalmol

2019;103:711-720.

\section{ABSTRACT}

Progressive cone and cone-rod dystrophies are a clinically and genetically heterogeneous group of inherited retinal diseases characterised by cone photoreceptor degeneration, which may be followed by subsequent rod photoreceptor loss. These disorders typically present with progressive loss of central vision, colour vision disturbance and photophobia. Considerable progress has been made in elucidating the molecular genetics and genotype-phenotype correlations associated with these dystrophies, with mutations in at least 30 genes implicated in this group of disorders. We discuss the genetics, and clinical, psychophysical, electrophysiological and retinal imaging characteristics of cone and cone-rod dystrophies, focusing particularly on four of the most common disease-associated genes: GUCA1A, PRPH2, $A B C A 4$ and $R P G R$. Additionally, we briefly review the current management of these disorders and the prospects for novel therapies.

\section{INTRODUCTION}

Inherited retinal diseases (IRDs) are a large group of clinically and genetically heterogeneous conditions which constitute the leading cause of legal blindness in England and Wales among working-age adults, and the second most common in childhood. ${ }^{1}$ One subgroup of IRDs is the progressive cone dystrophies (CODs) and cone-rod dystrophies (CORDs), characterised by the primary degeneration of cone photoreceptors often with later rod involvement. Their estimated incidence ranges from 1 in 20 $000-100000 .^{23}$

Inherited disorders of cone function are classically divided into two subtypes: stationary ${ }^{4}$ and progressive. ${ }^{5}$ The stationary cone disorders (cone dysfunction syndromes) are congenital/early-infantile onset and give rise to purely cone dysfunction, whereas progressive cone dystrophies are of later onset and usually also involve rod photoreceptors. There may, however, be overlap as some forms of cone dysfunction syndrome, such as achromatopsia, ${ }^{467}$ are associated with limited progression over time in a minority of subjects.

Recent advances in molecular genetics, particularly next-generation sequencing (NGS), have greatly improved molecular diagnosis, as the underlying causative genes and mutations can be identified in a large proportion of patients with COD and CORD. Many of these genes encode proteins involved in photoreceptor structure, or the phototransduction cascade.

\section{PHOTORECEPTION AND THE PHOTOTRANSDUCTION CASCADE}

Rod photoreceptors contain rhodopsin photopigment, whereas cone photoreceptors contain one of three types of opsin: S-cone, M-cone or L-cone opsin. Disease-causing sequence variants in the genes encoding the latter two cone opsins (OPN1MW and OPN1LW, respectively) are implicated in X linked (XL) Bornholm eye disease and S-cone monochromatism. ${ }^{8}$ The latter disorder, although usually stationary, may show a progressive phenotype.

\section{Photoreceptor activation}

The first stage of phototransduction involves the light-induced activation of rhodopsin, in which its bound chromophore, 11-cis-retinal, is isomerised into all-trans-retinol (figure 1). ${ }^{9}$ The resulting conformational change allows rhodopsin to interact with transducin, a guanine nucleotide-binding protein, to trigger dissociation of its $\alpha$-subunit. In turn, the transducin $\alpha$-subunit activates cyclic guanosine monophosphate (cGMP)-phosphodiesterase (PDE) by removal of its inhibitory $\gamma$-subunits, thus reducing intracellular cGMP levels and inducing closure of cGMP-gated (CNG) cation channels. As the membrane $\mathrm{Na}^{+}-\mathrm{Ca}^{2+}-\mathrm{K}^{+}$exchanger channels remain active with ongoing ion exchange, the $\mathrm{CNG}$ channel closure leads to decreased intracellular cation levels and cell hyperpolarisation. ${ }^{10}$

Recessive variants in PDE6C and PDE6H, which encode the cone photoreceptor PDE $\alpha$-subunits and $\gamma$-subunits, respectively, are associated with autosomal recessive (AR)-COD. ${ }^{11}{ }^{12}$ Similarly, disease-causing variants in CNGA3 and CNGB3, which encode the CNG channel $\alpha$-subunit and ß-subunit, respectively, impair the CNG-mediated dark current that is normally modulated by light inputs. These sequence variants typically result in achromatopsia, although some variants may cause an AR-COD or CORD phenotype. ${ }^{6713}$

\section{Photoreceptor deactivation}

Following their activation, the phototransduction molecules enter a refractory period during which intracellular mechanisms return the photoreceptor to its dark state. The activated photopigment (rhodopsin) is phosphorylated by a 

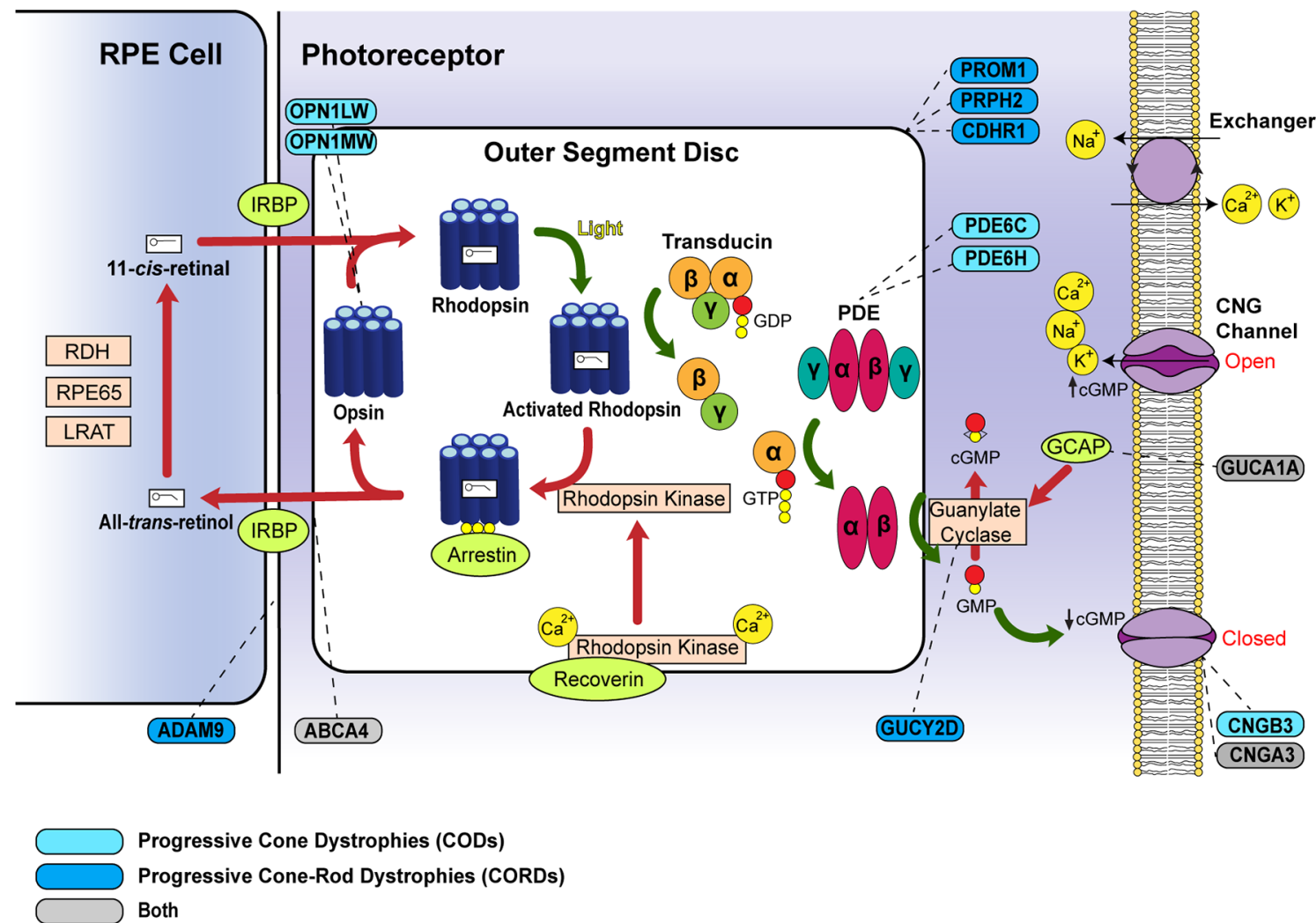

Figure 1 Schematic diagram of the phototransduction cascade including genes related to progressive cone dystrophies (CODs) and cone-rod dystrophies (CORDs). The cascade is triggered in the photoreceptor disc membrane by light-induced activation of rhodopsin, which subsequently activates transducin and phosphodiesterase (PDE). Activated PDE leads to cGMP hydrolysis to GMP. The decreased intracellular cGMP levels induce cation channel closure in the outer segment membrane and result in photoreceptor hyperpolarisation. Steps leading to photoreceptor activation are denoted by green arrows, whereas those causing photoreceptor deactivation are marked by red arrows. Corresponding genes for the proteins associated with CODs/CORDs are indicated by dashed lines. COD-associated genes are coloured in light blue and CORD-associated genes in dark blue, while those that can cause either phenotype are in grey. CGMP, cyclic GMP; CNG, cyclic nucleotide-gated; GCAP, guanylate cyclase-activating protein; GDP, guanosine diphosphate; GMP, guanosine monophosphate; GTP, guanosine triphosphate; IRBP, interphotoreceptor retinoid-binding protein; LRAT, lecithin retinol acyltransferase; PDE, phosphodiesterase; $\mathrm{RDH}$, retinol dehydrogenase; RPE65, retinal pigment epithelium $65 \mathrm{kDa}$.

G-protein-coupled receptor kinase (rhodopsin kinase), after which it is preferentially bound and inactivated by arrestin. Another mechanism by which the photoreceptor returns to its basal state is via retinal guanylate cyclase (RetGC1, encoded by GUCY2D). This enzyme replenishes intracellular cGMP levels following activation by $\mathrm{Ca}^{2+}$-sensitive guanylate cyclase-activating proteins (GCAPs). The interaction of intracellular cGMP with membrane CNG channels mediates an open configuration in the latter, causing cation influx and membrane depolarisation. ${ }^{9}$

Autosomal dominant (AD)-COD and CORD phenotypes may be associated with variants in GUCA1A, which encodes the GCAP1 protein. The common p.(Tyr99 Cys) substitution is most associated with COD, ${ }^{14}$ but also with a range of other phenotypes, ${ }^{15}$ while the p.(Pro50Leu) variant often results in CORD. ${ }^{16}$ In contrast, GUCY2D variants are arguably associated with less phenotypic variability and result in $\mathrm{AD}$-CORD. ${ }^{17}$ The degree of rod involvement is, however, milder in GUCY2D single-variant families compared to those with complex sequence variants. ${ }^{13} 19$

\section{GENETIC AND CLINICAL CHARACTERISTICS OF COD AND CORD}

Molecular pathology

To date, mutations in 32 genes are reported to cause COD or CORD (table 1). There are currently 6 identified genes that predominantly cause COD and 22 that lead to CORD. However, there is considerable overlap with the majority of genes associated with rod involvement over time.

The proteins encoded by these genes perform a diverse range of functions in the photoreceptor, including phototransduction (as outlined above), outer segment (OS) morphogenesis (CDHR1, PROM1, PRPH2), ${ }^{20-22}$ intraflagellar transport (RAB28, RPGR $)^{23} 24$ and neurotransmitter release (RIMS1, UNC119). ${ }^{2526}$

In order to assess the relative contribution of each gene in each mode of inheritance (AD, AR and $\mathrm{XL}$ ), all publicly available literature on CODs and CORDs (PubMed search November 2018) in which unrelated probands were genetically investigated was analysed (online supplementary tables 1-3). The number of patients with COD/CORD secondary to disease-causing variants in a given gene was counted as a percentage of the total number of $\mathrm{COD} / \mathrm{CORD}$ in each cohort. Using this approach, we estimated that the disease-causing gene is identified in $56.3 \%$ of patients with COD/CORD, while the remaining $43.7 \%$ of cases are unsolved (figure $2 \mathrm{~A}$ ). The majority of molecularly defined disease is recessively inherited, with variants in the $A B C A 4$ gene accounting for $62.2 \%$ of AR-COD/CORD cases. In AD-inherited and XL-inherited cases, variants in GUCY2D (34.6\%) and RPGR (73.0\%) constitute the most prevalent monogenic causes of disease, respectively, among currently identified genes (figure 2B-D). 
Table 1 Summary of the identified disease-causing genes in progressive cone and cone-rod dystrophy

\begin{tabular}{|c|c|c|c|c|c|}
\hline $\begin{array}{l}\text { Classification and } \\
\text { inheritance pattern }\end{array}$ & $\begin{array}{l}\text { Gene } \\
\text { abbreviation }\end{array}$ & Gene name & Gene locus & Potential function & $\begin{array}{l}\text { Other associated } \\
\text { phenotypes } \\
\text { (OMIM) }\end{array}$ \\
\hline \multicolumn{6}{|c|}{ Progressive cone dystrophy (COD) } \\
\hline \multirow[t]{4}{*}{ Autosomal recessive } & CACNA2D4 & Voltage-dependent calcium channel alpha-2/delta-4 & $12 \mathrm{p} 13.33$ & Neurotransmitter release & - \\
\hline & CNGB3 & Cyclic nucleotide-gated channel beta-3 & $8 q 21.3$ & Phototransduction & $\mathrm{ACHM}$ \\
\hline & PDE6C & Cone-specific phosphodiesterase alpha subunit & $10 q 23.33$ & Phototransduction & $\mathrm{ACHM}$ \\
\hline & PDE6H & Cone-specific phosphodiesterase gamma subunit & $12 \mathrm{p} 12.3$ & Phototransduction & $\mathrm{ACHM}$ \\
\hline \multirow[t]{2}{*}{$\mathrm{X}$ linked } & OPN1LW & Long-wave-sensitive opsin 1 & $\mathrm{Xq28}$ & Phototransduction & BCM, BED \\
\hline & OPN1MW & Medium-wave-sensitive opsin 1 & Xq28 & Phototransduction & BCM, BED \\
\hline \multicolumn{6}{|c|}{ Progressive cone-rod dystrophy (CORD) } \\
\hline \multirow[t]{9}{*}{ Autosomal dominant } & AIPL1 & Aryl-hydrocarbon-interacting protein-like 1 & $17 p 13.2$ & Tissue development & LCA, RP \\
\hline & CRX & Cone-rod homeobox-containing gene & $19 q 13.33$ & Tissue development & LCA, MD \\
\hline & GUCY2D & Guanylate cyclase 2D & $17 p 13.1$ & Photoreceptor recovery & CACD, LCA \\
\hline & PITPNM3 & $\begin{array}{l}\text { Membrane-associated phosphatidylinositol transfer } \\
\text { protein } 3\end{array}$ & 17p13.2-p13.1 & Tyrosine kinase signalling & - \\
\hline & PROM1 & Prominin 1 & $4 p 15.32$ & Outer segment morphogenesis & $\mathrm{MD}, \mathrm{RP}$ \\
\hline & PRPH2 & Peripherin 2 & $6 \mathrm{p} 21.1$ & Outer segment morphogenesis & CACD, LCA, MD, RP \\
\hline & RAX2 & Retina and anterior neural fold homeobox 2 & 19p13.3 & Tissue development & - \\
\hline & RIMS1 & Protein regulating synaptic membrane exocytosis 1 & $6 q 13$ & Neurotransmitter release & $\mathrm{RP}$ \\
\hline & UNC119 & Human homologue of C.elegans UNC119 protein & $17 q 11.2$ & Neurotransmitter release & - \\
\hline \multirow[t]{12}{*}{ Autosomal recessive } & ADAM9 & A disintegrin and metalloproteinase domain 9 & $8 p 11.22$ & Outer segment-RPE junction & - \\
\hline & C210RF2 & Chromosome 21 open reading frame 2 & $21 \mathrm{q} 22.3$ & Ciliogenesis & - \\
\hline & C80RF37 & Chromosome eight open reading frame 37 & $8 q 22.1$ & Unknown & $\mathrm{RP}$ \\
\hline & CDHR1 & Cadherin-related family member 1 & $10 \mathrm{q} 23.1$ & Outer segment morphogenesis & $\mathrm{RP}$ \\
\hline & CEP78 & Centrosomal protein 78-kD & $9 q 21.2$ & Ciliogenesis & - \\
\hline & CERKL & Ceramide kinase-like & $2 q 31.3$ & Photoreceptor survival & $\mathrm{RP}$ \\
\hline & KCNV2 & Potassium voltage-gated channel subfamily V 2 & $9 p 24.2$ & Unknown & - \\
\hline & POC1B & Proteome of the centriole $1 \mathrm{~B}$ & $12 q 21.33$ & Intraflagellar transport & - \\
\hline & RAB28 & Ras-associated protein 28 & $4 p 15.33$ & Intraflagellar transport & - \\
\hline & RPGRIP1 & Retinitis pigmentosa GTPase regulator protein 1 & $14 q 11.2$ & Intracellular trafficking & LCA \\
\hline & SEMA4A & Semaphorin $4 \mathrm{~A}$ & $1 q 22$ & Tissue development & $\mathrm{RP}$ \\
\hline & TTLL5 & Tubulin tyrosine ligase-like family member 5 & $14 \mathrm{q} 24.3$ & Steroid receptor signalling & - \\
\hline $\mathrm{X}$ linked & CACNA1F & Voltage-dependent calcium channel alpha-1F & Xp11.23 & Neurotransmitter release & AIED, CSNB \\
\hline \multicolumn{6}{|l|}{ Both } \\
\hline Autosomal dominant & GUCA1A & Guanylate cyclase activator $1 \mathrm{~A}$ & $6 p 21.1$ & Photoreceptor recovery & - \\
\hline \multirow[t]{2}{*}{ Autosomal recessive } & ABCA4 & ATP-binding cassette subfamily A member 4 & $1 \mathrm{p} 22.1$ & Retinoid cycle & MD, STGD \\
\hline & CNGA3 & Cyclic nucleotide-gated channel alpha-3 & $2 q 11.2$ & Phototransduction & ACHM \\
\hline $\mathrm{X}$ linked & RPGR & Retinitis pigmentosa GTPase regulator & Xp11.4 & Intraflagellar transport & $\mathrm{MD}, \mathrm{RP}$ \\
\hline
\end{tabular}

ACHM, achromatopsia; AIED, Aland Island eye disease; BCM, blue cone monochromacy; BED, Bornholm eye disease; CACD, central areolar choroidal dystrophy; CSNB, congenital stationary night blindness; LCA, Leber congenital amaurosis; MD, macular dystrophy; OMIM, online Mendelian inheritance in man; RP, retinitis pigmentosa; RPE, retinal pigment epithelium; STGD, Stargardt disease.

\section{Clinical presentation}

COD presents with loss of central vision, photophobia and colour vision disturbance. Since cone function is usually initially normal, nystagmus is often absent. COD is distinguishable from CORD by the absence of early nyctalopia, which occurs in the latter due to concomitant rod degeneration. However, the majority of patients with COD develop rod dysfunction or loss as the disease progresses. ${ }^{4}$ In most cases, CODs and CORDs affect colour discrimination in all three colour axes due to parallel cone degeneration of the three opsin subtypes. Kellner et $a l^{27}$ and Went $e t a l^{28}$ present notable exceptions to this with preferential degeneration of L-opsin and S-opsin cones, respectively, thereby causing a protan or tritan defect.

A longitudinal study by Thiadens $e$ t $a l^{29}$ demonstrated earlier symptomatic onset in CORD than COD (12 vs 16 years), as well as a more severe disease course based on psychophysical testing. Visual acuity in more than half of patients with CORD $(n=83)$ deteriorated to legal blindness by the age of 23 years, compared with 48 years in $\operatorname{COD~}(n=98)$, although there was a large degree of individual variability and overlap.

\section{Clinical investigation}

Psychophysical assessment

Reduced visual acuity is the earliest manifestation of $\mathrm{COD} /$ CORD, generally occurring in the first decade of life and not significantly improved by spectacle wear. ${ }^{2}$ The presence of an isolated central scotoma on visual field testing is typical in patients with COD, but cannot be used alone for discriminating the diagnosis from CORD. A significant proportion of patients with CORD retain peripheral vision at the time of disease onset, and develop a peripheral scotoma up to 10 years later. ${ }^{29}$ In general, CODs and CORDs lead to marked visual loss at an 
A Mode of Inheritance

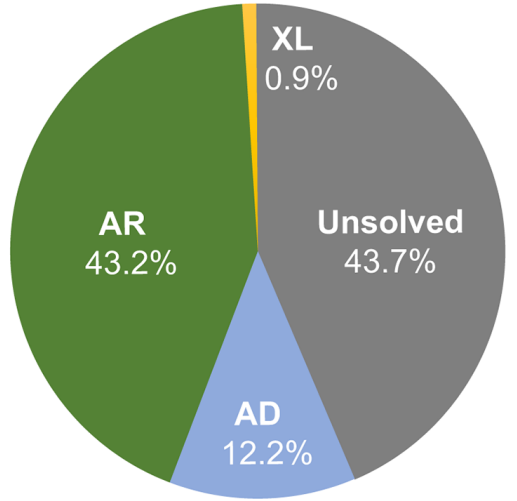

C Autosomal Recessive (AR)

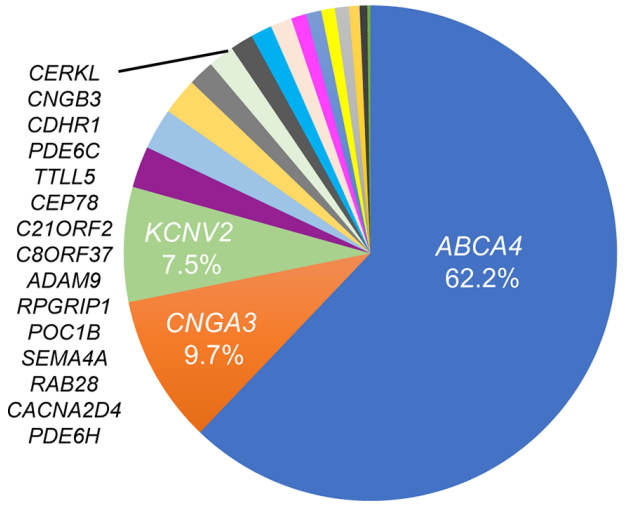

B Autosomal Dominant (AD)

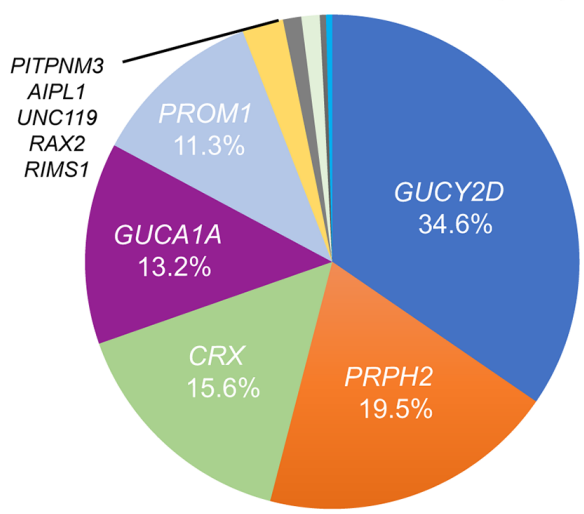

D

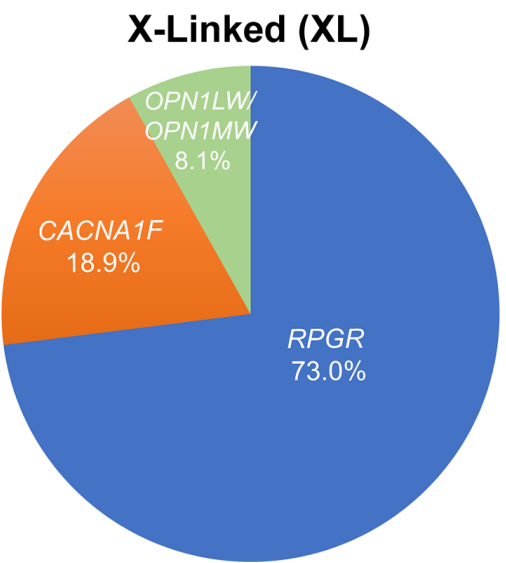

Figure 2 Frequency of disease-causing genetic variants leading to progressive cone dystrophies (CODs) and cone-rod dystrophies (CORDs), using studies with clearly indicated cohort sizes (listed in online supplementary tables 1-3). (A) Prevalence of the mode of inheritance for CODs and CORDs. The underlying disease-causing gene is identified in 56.3\% of COD/CORD cases, of which most (43.2\%) are of autosomal recessive (AR) inheritance. This is followed by autosomal dominant $(A D)$ inheritance (12.2\%) and $X$ linked $(X L)$ inheritance $(0.9 \%)$ patterns. The remaining $43.7 \%$ of patients are unsolved with regard to molecular causation. (B) AD inheritance of CODs and CORDs. Mutations in 10 genes are currently associated with AD-COD/ CORD, over 75\% of which are accounted for by GUCY2D, PRPH2, CRX and GUCA1A. (C) AR inheritance of CODs and CORDs. Mutations in 18 genes are currently associated with AR-COD/CORD, of which $A B C A 4$ is by far the most common (62.2\%). (D) XL inheritance of CODs and CORDs. Mutations in 4 genes are currently associated with XL-COD/CORD, of which RPGR accounts for $73.0 \%$ of cases.

earlier age than retinitis pigmentosa (RP), a rod-cone dystrophy, and are thus arguably more severe conditions. ${ }^{2}$

\section{Electroretinography}

The earliest electroretinography (ERG) finding in COD/CORD is a delayed $30 \mathrm{~Hz}$ flicker ERG implicit time, which selectively assesses cone response. ${ }^{2}$ This is followed by deterioration of the $30 \mathrm{~Hz}$ flicker ERG amplitude, and reduced a-wave and b-wave amplitudes of the single flash photopic ERG. Scotopic function is preserved in early disease, but is usually affected in late disease. In corroboration with psychophysical findings, longitudinal ERG in CORD shows a faster rate of cone functional decline than in patients with COD. ${ }^{30}$

In one disorder, KCNV2-associated retinopathy, the ERG findings are characteristic and diagnostic; there is an abnormal cone ERG with 'supernormal' rod responses. ${ }^{31}$ Despite its nomenclature, this disorder is not associated with enhanced rod function measured psychophysically. ${ }^{32}$

\section{Retinal imaging}

Funduscopy in COD classically reveals a bull's eye maculopathy, but may more often identify bilateral and relatively symmetrical retinal pigment epithelium (RPE) disturbance/atrophy with progression over time. ${ }^{5}$ Peripheral RPE atrophy and bone spicule pigmentation are observed in advanced stages of CORD, whereas the retinal periphery is typically normal in CODs due to rod preservation. White flecks have, however, been described at the level of the RPE, ${ }^{33}$ with such cases likely to develop rod dysfunction over time. Other reported findings include a dark choroid sign on fluorescein angiography ${ }^{34}$ and a tapetal-like sheen in XL-CORD. ${ }^{35}$

Fundus autofluorescence (FAF) and optical coherence tomography (OCT) imaging have greatly improved the characterisation of IRDs. Using wide-field FAF, Oishi et $a l^{36}$ demonstrated an association between abnormal AF and the severity of functional impairment in COD/CORD, also correlating the extent of reduced autofluorescence with symptom duration. ${ }^{37}$ These findings are substantiated by their correlation with ERG abnormalities. ${ }^{38}$ On OCT, an absent interdigitation zone is an early occurrence in COD and CORD, which is a band representing the interaction between apical processes of the RPE and the photoreceptor OS. Progressive disruption and loss of the ellipsoid zone (EZ) is another notable finding, which corresponds to the ellipsoid portion of the photoreceptor inner segment. ${ }^{3940}$ Reduction in EZ band reflectance may also be seen in some CODs and CORDs. ${ }^{41}{ }^{42}$ In advanced disease, outer retinal atrophy including the RPE is observed. 
Exploration of phenotypic diversity in COD and CORD has been transformed over the last decade with the application of adaptive optics (AO) in retinal imaging. ${ }^{42} 43$ This technique has enabled imaging of the human retina at a cellular resolution by real-time measurement and correction of individual optical aberrations. Usually integrated with scanning light ophthalmoscopy (SLO), AO imaging has been used to characterise and quantify the central macular photoreceptor mosaic in multiple COD/CORD cohorts. In GUCY2D-associated AD-CORD, ${ }^{44}$ for example, residual cone inner segments were visualised at the fovea which were undetected by other imaging modalities. Longitudinal AO imaging thus offers utility in measuring the rate of cone cell loss in progressive disease with precision. ${ }^{45}$

\section{SPECIFIC EXAMPLES OF COD AND CORD \\ Autosomal dominant GUCA1A-associated COD/CORD \\ Molecular genetics}

GUCA1A is a four-exon gene encoding GCAP1, which is required for RetGC activation and cGMP regeneration. ${ }^{46}$ Since it requires regulation in a $\mathrm{Ca}^{2+}$-dependent manner, GCAP1 contains three $\mathrm{Ca}^{2+}$-binding EF-hand motifs, structural alterations to which occur in most disease-causing GUCA1A sequence variants. ${ }^{47}$ These include the gain-in-function variants p.(Tyr99Cys), ${ }^{16}$ p.(Glu155Gly) ${ }^{48}$ and p.(Asp100Gly). ${ }^{49}$ These result in persistent stimulation of RetGC, excess cGMP levels in the dark and photoreceptor apoptosis secondary to $\mathrm{Ca}^{2+}$ dysregulation. ${ }^{50} 51$
The phenotypic variability in patients harbouring identical GUCA1A mutations is noteworthy. In an intrafamilial example, Michaelides et $a l^{15}$ demonstrated the p.(Tyr99Cys) missense variant resulting in three different dominantly inherited phenotypes in a non-consanguineous British family: COD, CORD and isolated macular dystrophy (figure 3). Similarly, another study identified a p.(Arg120Leu) substitution as the cause of clinically heterogeneous macular dystrophy in a Chinese family, with whole exome sequencing (WES) excluding mutations in other IRD genes. ${ }^{52}$

\section{Clinical assessment}

Symptomatic onset usually occurs between the second and third decade with reduced central vision, photophobia and generalised dyschromatopsia. ERG studies characteristically show reduced cone single-flash and flicker amplitudes with a normal implicit time (an unusual finding in generalised retinal disease). Rod function typically remains normal, although dysfunction may occur over time in COD or be reduced at presentation in CORD. The relative preservation of rod responses in most GUCA1A-associated progressive retinal dystrophies is attributed to greater GCAP1 expression in cones. ${ }^{53}$

Figure 3 Longitudinal analysis of phenotypically heterogeneous GUCA1A-associated retinopathy (p.(Tyr99Cys) substitution). Fundus autofluorescence (FAF) and optical coherence tomography (OCT) imaging in unrelated subjects (A and B) harbouring the p.(Tyr99Cys) (Y99C) variant in GUCA1A. The left column shows FAF at baseline, and the right column that at the same location on follow-up. Red arrowheads point to the transfoveal OCT line scan at the location denoted by red dashes on FAF. Subject (A): presented with cone dystrophy (A-I baseline) which progressed over a follow-up period of 8 years (A-I follow-up). High magnification $(\times 5)$ of the same location in the foveal centre (A-II) at baseline and follow-up (left and right, respectively) shows a greater degree of disruption to the ellipsoid zone (EZ) in the latter (black arrows). Subject (B): presented with isolated macular dystrophy which progressed over a 4-year follow-up period. FAF and OCT scans are in the same scale; scale bar=200 $\mu \mathrm{m}$. 


\section{Retinal imaging}

Funduscopy findings are varied, ranging from mild RPE disturbance to extensive macular atrophy. FAF is useful in investigating macular abnormalities, although both areas of hypoautofluorescence and hyperautofluorescence have correlated with retinal atrophy; an increased signal at the fovea may be seen in early disease. ${ }^{5552}$ AOSLO has identified cellular variability between two related patients harbouring a single 428delTinsACAC insertion/ deletion variant. ${ }^{54}$ Despite similar clinical findings, they significantly differed in the degree of photoreceptor mosaic disruption, suggesting that other genetic (or environmental) factors influence the effect of the primary disease-causing variant.

\section{Autosomal dominant PRPH2-associated CORD Molecular genetics}

$P R P H 2$ is a five-exon gene encoding peripherin-2, a cell surface glycoprotein in the OS with an essential role in disc morphogenesis. ${ }^{55}$ Interactions of peripherin-2 with ROM1 and glutamic acid-rich domains of CNG channels support its function in disc stabilisation and maintenance of rim curvature. ${ }^{21}$ CORD-associated variants in $P R P H 2$ can be attributed to the region encoding the second intradiscal loop between its four transmembrane components. This contains cysteine residues essential for intraprotein folding and interprotein interactions. ${ }^{56}$ Identified missense variants in this region include p.(Asn244His), ${ }^{57}$ p.(Val200Glu) ${ }^{58}$ and p.(Arg172Trp). ${ }^{59}$

Families harbouring the p.(Asn244His) or p.(Val200Glu) variant present with early central RPE atrophy that advances peripherally on disease progression, with little intrafamilial variability. ${ }^{57} 58$ In contrast, clinical phenotypes associated with p.(Arg172Trp) can vary substantially, ranging from non-penetrance to severe CORD (figure $4 \mathrm{~A}, \mathrm{~B}) .{ }^{59}$ In addition to CORD, the p.(Arg172Trp) substitution is associated with other phenotypes, including RP, macular dystrophy and central areolar choroidal dystrophy. ${ }^{59-62}$

\section{Clinical assessment}

PRPH2-associated CORD usually presents in the second to third decade with reduced central vision, photophobia and nyctalopia. Certain genotype-phenotype correlations have been observed, including p.(Arg172Trp)-PRPH2 retinopathy being associated with faster loss of visual acuity than the p.(Arg172Gln) variant. ${ }^{62}$

\section{Retinal imaging}

Patients vary considerably in their funduscopic appearance, ranging from a bull's eye maculopathy (figure 4A) to macular atrophy (figure 4B). However, FAF in PRPH2-associated disease, including the p.(Arg172Trp) variant, demonstrates a characteristic speckled macular appearance in most patients (figure 4A, III-IV). ${ }^{59}$ AOSLO imaging in p.(Arg172Trp)-associated CORD revealed increased cone spacing throughout the macula with corresponding loss of outer retinal structures on OCT. ${ }^{63}$ However, intrafamilial analysis of p.(Arg172Gln)-associated disease has shown marked variability, similar to that seen in GUCA1A-associated CORD; one family member had a completely normal photoreceptor mosaic, whereas two others had variable parafoveal cone loss. ${ }^{64}$
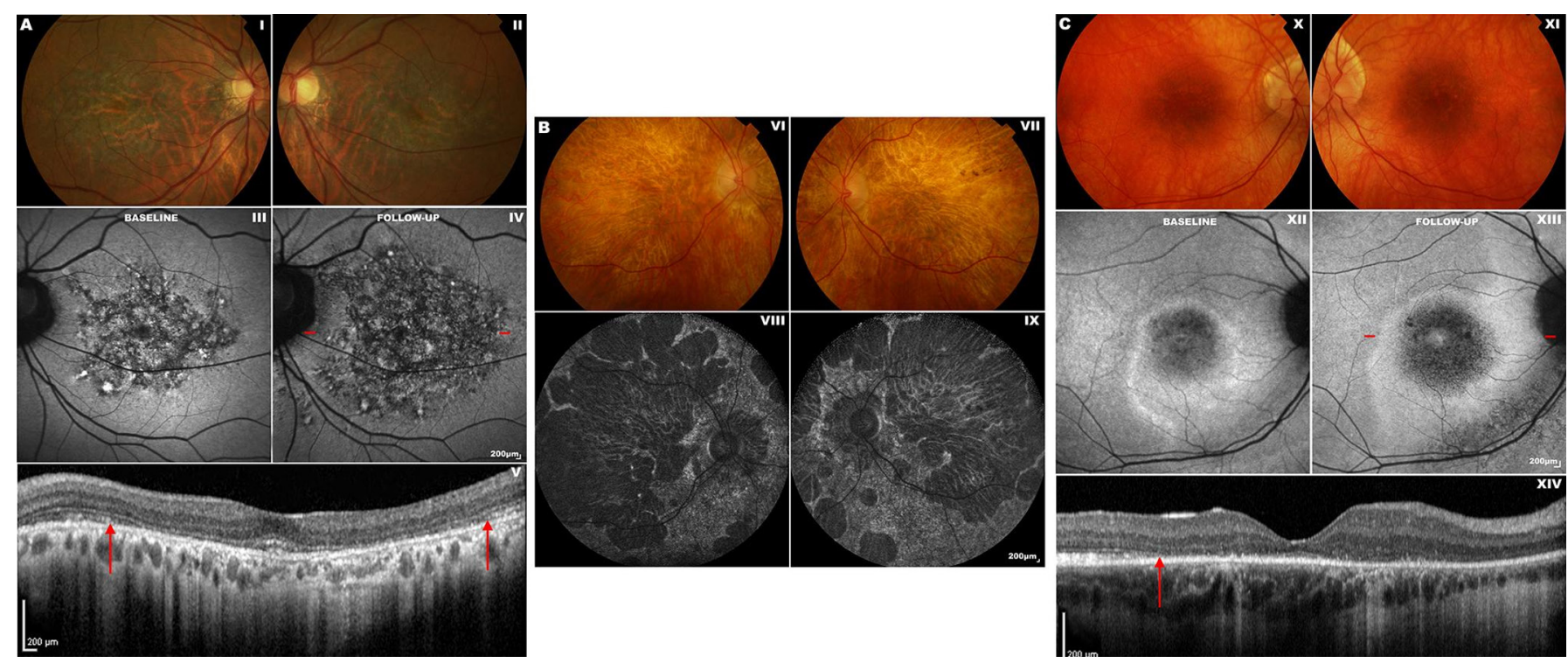

Figure 4 Fundus photography, fundus autofluorescence (FAF) and optical coherence tomography (OCT) imaging in unrelated patients with PRPH2associated and $R P G R$-associated cone-rod dystrophy (CORD). Subjects (A) and (B) possess the p.(Arg172Trp) (R172W) variant in PRPH2. Subject (C) possesses the c.2847_2848delinsCT, p.(E950*) variant in RPGR. Subject (A): fundus photographs of both eyes (I-II) show bilateral bull's eye maculopathy-like retinal pigment epithelial (RPE) changes. FAF imaging of the left eye at baseline (III) and on 11-year follow-up (IV) displays a florid speckled appearance with areas of increased and decreased macular autofluorescence. The area of affected retina is substantially increased in (IV), with red dashes denoting the location of the OCT scan (V). The inner segment ellipsoid photoreceptor-derived layer (the ellipsoid zone, EZ) between the red arrows in (V) is absent. Subject (B): fundus photographs of both eyes (VI-VII) showing marked bilateral macular atrophy, peripheral areas of RPE atrophy and pigmentation. Corresponding FAF images are shown in (VIII-IX). This patient has severe CORD with an acuity of counting fingers bilaterally and constricted peripheral visual fields. Subject (C): fundus photographs of both eyes (X-XI) showing bilateral macular atrophy, which corresponds to the areas of decreased macular autofluorescence. FAF imaging of the right eye at baseline (XII) and on 7-year follow-up (XIII) shows an increase in the hypoautofluorescent area and surrounding hyperautofluorescent ring ${ }^{88}$ The red dashes in (XIII) denote the location of the OCT scan (XIV), in which the temporal border of the photoreceptor layer is marked with a red arrow. At its nasal aspect, the integrity of the photoreceptor layer is disrupted. 


\section{Autosomal recessive $A B C A 4$-associated COD/CORD}

Molecular genetics

$A B C A 4$ is a 50 -exon gene that encodes a retina-specific ATP-dependent cassette transporter located in the curved rim of the OS disc membrane. ${ }^{65}$ This protein has an essential role in the removal of $\mathrm{N}$-retinylidene-phosphatidylethanolamine (PE) from the luminal to cytoplasmic aspect of the OS disc membrane, which is produced from the reaction of $\mathrm{PE}$ with excess chromophores. ${ }^{9}$ If not exported and dissociated, $\mathrm{N}$-retinylidene-PE can accumulate in the OS to form the toxic fluorophore, $\mathrm{N}$-retinylidene- $\mathrm{N}$-retinylethanolamine (A2E), a component of lipofuscin.

$A B C A 4$ is one of the most common genetic causes of IRD and is associated with vast phenotypic variability. Over 1000 diseasecausing variants in $A B C A 4$ have been identified to date, with the resulting phenotype varying between COD, CORD and Stargardt disease (STGD). ${ }^{66}$ In general, biallelic null variants are more commonly associated with severe and earlier onset CORD and childhood-onset STGD, ${ }^{67} 68$ whereas biallelic missense variants are associated with milder disease such as later onset and foveal-sparing forms of STGD. ${ }^{69}{ }^{70}$ Functional outcome is dependent on both the variant itself and interaction with other $A B C A 4$ variants (and other genetic modifiers). For example, p.(Gly1961Glu) in the homozygous state typically results in a milder phenotype, ${ }^{71}$ but can be associated with more severe disease when combined with other variants. ${ }^{72}$

\section{Clinical assessment}

Symptomatic onset usually occurs in childhood with a central scotoma and rapidly progressing macular atrophy. ${ }^{68}$ The majority of patients have rod involvement at presentation (CORD), which is associated with a worse prognosis. ${ }^{73}$

\section{Retinal imaging}

Funduscopy may initially reveal a normal fundus or mild retinal abnormalities (such as loss of foveal reflex), as peripheral degenerative changes occur in later disease. ${ }^{74}$ Diagnosis can therefore be delayed unless FAF or OCT imaging is undertaken. ${ }^{68}$ FAF findings include a bull's eye maculopathy-like appearance with yellow-white retinal flecks and increasing macular atrophy over time. ${ }^{73} 75$ OCT reveals loss of outer retinal architecture at the central macula. ${ }^{76}$ Longitudinal increase in abnormal AF regions correlates with both visual functional decline and abnormal cone spacing on AOSLO. ${ }^{77}$ However, cone mosaic abnormalities are known to precede abnormal psychophysical testing and FAF (figure 5$).^{78}$

\section{X linked RPGR-associated COD/CORD \\ Molecular genetics}

$R P G R$ is a 19-exon gene that gives rise to two alternatively spliced retinal isoforms, encoded by exons 1-19 and 1-15 (+ part of intron 15), respectively. ${ }^{79}$ The latter isoform, also known as exon open reading frame 15 (ORF15), is the most highly expressed retinal variant and a mutational hot spot that accounts for most XL COD and CORD cases. ${ }^{50}$ The function of the C-terminal ORF15 sequence requires further elucidation, but is implicated in intraflagellar protein transport in view of its localisation at the photoreceptor connecting cilium. ${ }^{24}$

Most disease-causing variants in $R P G R$ result in $\mathrm{RP}^{81}$ but those leading to $\mathrm{COD} / \mathrm{CORD}$ are preferentially sequestered at the 3 ' end of the ORF15 region. ${ }^{82}$ In keeping with the majority of IRD, identical intrafamilial sequence variants in RPGR may lead to distinctly different phenotypes. ${ }^{83}$ This is exemplified by a Chinese family harbouring a 2403_04delAG deletion that resulted in both XL-RP and XL-CORD in affected men, reaffirming the importance of disease-modifying factors. ${ }^{84}$

\section{Clinical assessment}

$R P G R$-associated CORD is characterised by central visual loss, mild photophobia and myopia, and presents in the second to fourth decade in affected men. ${ }^{85} \mathrm{~A}$ longitudinal study reported significantly higher levels of legal blindness among RPGR-associated COD/CORD compared with RPGR-associated RP by the age of 40 years; high myopia was predictive of faster visual decline in this study. ${ }^{86}$

\section{Retinal imaging}

FAF imaging often reveals parafoveal rings of increased signal in $R P G R$-associated COD/CORD. Unlike RP, these increase in size with disease progression (figure 4C, XII-XIII) and are inversely related to ERG amplitude. ${ }^{87} 88$ They are, therefore, a potential endpoint in clinical trials.

\section{MANAGEMENT OF COD AND CORD}

At present, there are no proven treatments for COD/CORD that halt progression or restore lost vision. Current management consists of symptomatic alleviation, including refractive correction, use of tinted spectacles/contact lenses for photophobia and low vision aids. An accurate diagnosis using molecular genetics is an important step to facilitate genetic counselling, advice on prognosis and participation in anticipated clinical trials. ${ }^{89}$

Patients with specific forms of COD/CORD can be advised to adopt strategies to try to slow degeneration, based on a knowledge of gene function or investigation of animal models. In GUCA1A-associated retinopathy, sleeping with the lights on is advocated by some clinicians for preventing accumulation of cGMP, which otherwise occurs at night and causes photoreceptor damage. In contrast, light avoidance using tinted spectacles may confer benefit in $A B C A 4$-associated retinopathy by inhibiting $\mathrm{A} 2 \mathrm{E}$ production, ${ }^{90}$ which produces DNA-damaging epoxides. ${ }^{91}$ Vitamin A should also be avoided in ABCA4-associated retinopathy as it may enhance $\mathrm{A} 2 \mathrm{E}$ production and, therefore, disease progression. $^{92}$

Recent evidence has demonstrated the potential of gene therapy for long-term improvement in COD/CORD visual outcomes. Animal models of disease (murine and canine) in GUCA1A, ${ }^{93}$ PRPH $2,{ }^{94} A B C A 4{ }^{95}$ and $R P G R{ }^{96}$ have shown significant increase in photoreceptor survival following gene-based therapies. Gene therapy encompasses multiple techniques, including gene replacement, gene editing and gene silencing, with treatment choice dependent on whether the associated sequence variant(s) leads to a loss or gain in function. ${ }^{97}$ Human treatment trials of gene replacement therapy are already under way for retinal disease associated with mutations in $A B C A 4$ ( ClinicalTrials.gov identifier: NCT01367444) and RPGR (NCT03252847, NCT03116113 and NCT03316560), with results keenly awaited.

\section{LIMITATIONS OF GENETIC TESTING}

While therapeutic options are on the horizon for COD/CORD secondary to identified disease-causing genes, the outlook for patients without a molecular diagnosis is more limited. NGS-based approaches, typically using WES, have revolutionised genomic analysis, but not all pathogenic mutations can be detected. ${ }^{98}$ Complex changes, such as inversions, translocations and trinucleotide repeat expansions, are mostly undetected with WES, and variants in deep intronic or regulatory regions are not 

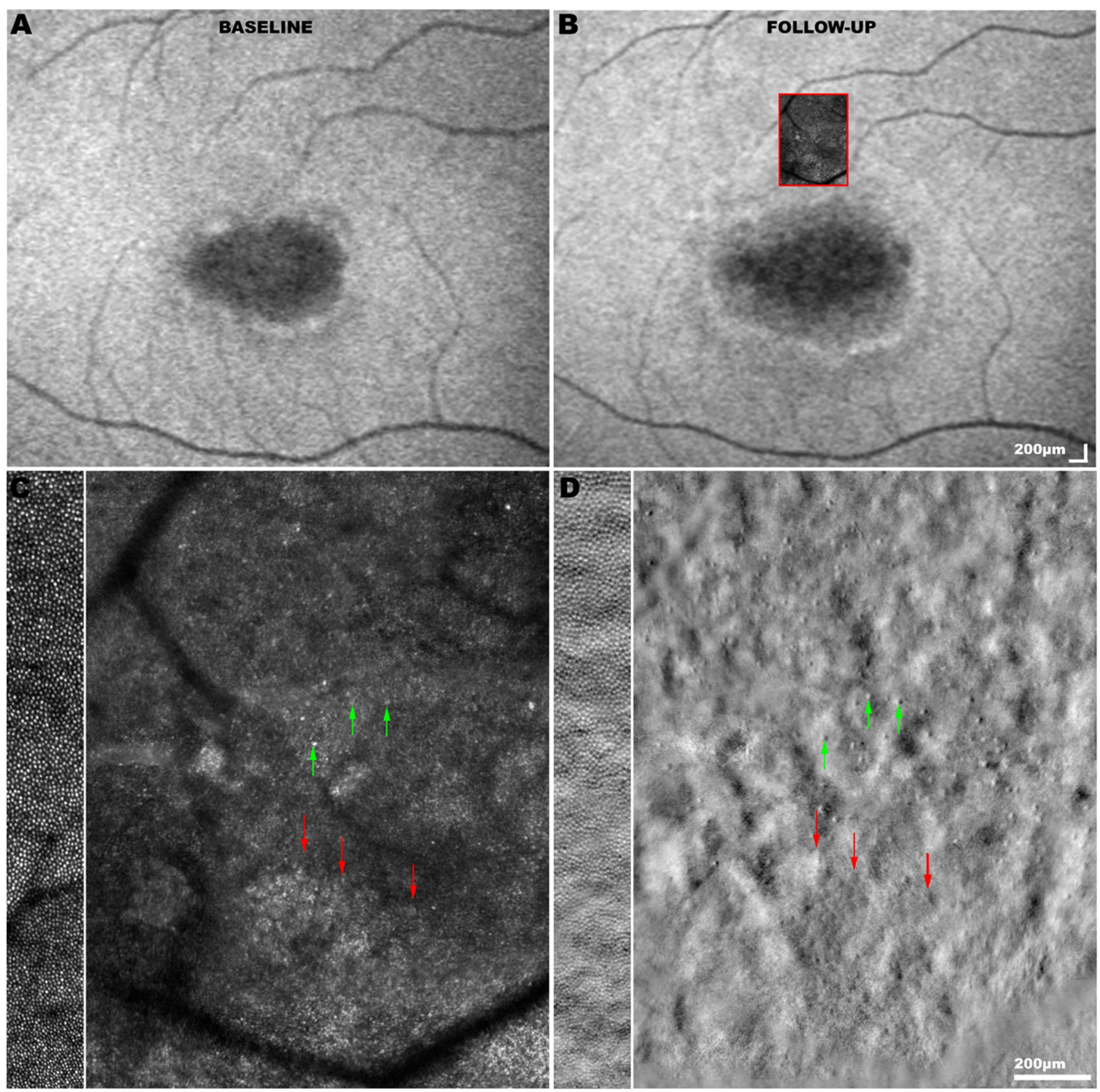

Figure 5 Fundus autofluorescence (FAF) and adaptive optics (AO) imaging in ABCA4-associated cone-rod dystrophy (CORD). (A) FAF image at baseline showing a central region of hypoautofluorescence surrounded by increased signal, and (B) aligned FAF image on 2-year follow-up demonstrating disease progression with an increased area of hypoautofluorescence. The red square signifies the superimposed adaptive optics scanning light ophthalmoscopy (AOSLO) montage acquired from that region on follow-up. (C) Confocal AOSLO image (photoreceptor outer segments) and (D) split-detection AOSLO image (photoreceptor inner segments) over the transition zone between less affected and more affected retina. For comparison, the side bars on the left show AOSLO images of an unaffected control at similar retinal eccentricity. Cone photoreceptors (green arrows) can be more reliably identified using split-detection imaging due to the poor wave guiding ability of outer segments in the confocal modality. The border of the transition zone (red arrows) in (C) corresponds to the presence of remnant cone inner segments in (D). The photoreceptor mosaic in CORD is disorganised, with altered regularity and reflectance compared to that of an unaffected eye. The number of cones is decreased in areas that appear healthy on FAF, demonstrating a disconnect between imaging modalities and supporting the utility of multimodal imaging. AOSLO images were acquired using a custom-built AOSLO housed at University College London (UCL) / Moorfields Eye Hospital (MEH); scale bar=200 $\mu \mathrm{m}$.

sequenced. Whole genome sequencing (WGS) offers a comprehensive alternative. However, there still remain a proportion of patients who are unsolved despite WGS, due to more complex genetic causes that remain challenging to identify and prove definitively. These include rearrangements and variants in distant promoter/enhancer regions. Over time, improvements in technology and understanding will gradually reduce the number of patients without a genetic diagnosis. ${ }^{99}$

\section{CONCLUDING REMARKS AND FUTURE PROSPECTS}

Advances in molecular genetic techniques, particularly NGS, have greatly simplified molecular diagnosis. It is hoped that the majority of patients will be able to have a precise molecular diagnosis in the future as the remaining causative genes and sequence variants in CODs and CORDs are identified. Similarly, advances in visual function testing and retinal imaging have improved knowledge of the relationship between genotype and phenotype, which is key to identifying treatment effects in clinical trials of novel therapies. The remaining challenge is to develop novel therapies that will slow degeneration or improve function, and it is encouraging that gene-based approaches to therapy are increasingly in clinical trial with the first Food and Drug Administration-approved gene therapy for LCA-RPE65 now available.

Contributors JSG conceived, wrote and provided critical revision of the manuscript. MG, AK and ATM provided critical revision of the manuscript. MM supervised and provided critical revision of the manuscript. Figures were produced by MG and JSG. 
Funding Supported by grants from the National Institute for Health Research, the Biomedical Research Centre at Moorfields Eye Hospital NHS Foundation Trust and the UCL Institute of Ophthalmology, Fight For Sight, Moorfields Eye Hospital Special Trustees, Moorfields Eye Charity, the Wellcome Trust (099173/Z/12/Z), Retina UK, and the Foundation Fighting Blindness (USA).

Competing interests None declared.

Patient consent for publication Not required.

Provenance and peer review Not commissioned; externally peer reviewed.

Open access This is an open access article distributed in accordance with the Creative Commons Attribution 4.0 Unported (CC BY 4.0) license, which permits others to copy, redistribute, remix, transform and build upon this work for any purpose, provided the original work is properly cited, a link to the licence is given, and indication of whether changes were made. See: http://creativecommons.org/ licenses/by/4.0

\section{REFERENCES}

1. Liew G, Michaelides M, Bunce C. A comparison of the causes of blindness certifications in England and Wales in working age adults (16-64 years), 1999-2000 with 2009-2010. BMJ Open 2014;4:e004015.

2. Hamel CP. Cone rod dystrophies. Orphanet J Rare Dis 2007;2:7.

3. Roosing $S$, Thiadens AA, Hoyng CB, et al. Causes and consequences of inherited cone disorders. Prog Retin Eye Res 2014;42:1-26.

4. Aboshiha J, Dubis AM, Carroll J, et al. The cone dysfunction syndromes. Br J Ophthalmol 2016;100:115-21.

5. Michaelides M, Hardcastle AJ, Hunt DM, et al. Progressive cone and cone-rod dystrophies: phenotypes and underlying molecular genetic basis. Surv Ophthalmol 2006;51:232-58.

6. Michaelides M, Aligianis IA, Ainsworth JR, et al. Progressive cone dystrophy associated with mutation in CNGB3. Invest Ophthalmo/ Vis Sci 2004;45:1975-82.

7. Thiadens AA, Roosing $S$, Collin RW, et al. Comprehensive analysis of the achromatopsia genes CNGA3 and CNGB3 in progressive cone dystrophy. Ophthalmology 2010;117:825-30.

8. Gardner JC, Michaelides M, Hardcastle AJ. Cone opsins, colour blindness and cone dystrophy: Genotype-phenotype correlations. S Afr Med J 2016;106(6 Suppl 1):75-8.

9. Molday RS, Moritz OL. Photoreceptors at a glance. J Cell Sci 2015;128:4039-45.

10. Palczewski K. Chemistry and biology of the initial steps in vision: the Friedenwald lecture. Invest Ophthalmol Vis Sci 2014;55:6651-72.

11. Piri N, Gao YQ, Danciger M, et al. A substitution of $G$ to $C$ in the cone cGMPphosphodiesterase gamma subunit gene found in a distinctive form of cone dystrophy. Ophthalmology 2005;112:159-66.

12. Thiadens $A A$, den Hollander Al, Roosing $S$, et al. Homozygosity mapping reveals PDE6C mutations in patients with early-onset cone photoreceptor disorders. Am J Hum Genet 2009;85:240-7.

13. Shaikh RS, Reuter P, Sisk RA, et al. Homozygous missense variant in the human CNGA3 channel causes cone-rod dystrophy. Eur J Hum Genet 2015;23:473-80.

14. Payne AM, Downes SM, Bessant DA, et al. A mutation in guanylate cyclase activator $1 \mathrm{~A}$ (GUCA1A) in an autosomal dominant cone dystrophy pedigree mapping to a new locus on chromosome 6p21.1. Hum Mol Genet 1998;7:273-7.

15. Michaelides M, Wilkie SE, Jenkins S, et al. Mutation in the gene GUCA1A, encoding guanylate cyclase-activating protein 1, causes cone, cone-rod, and macular dystrophy. Ophthalmology 2005;112:1442-7.

16. Downes SM, Holder GE, Fitzke FW, et al. Autosomal dominant cone and cone-rod dystrophy with mutations in the guanylate cyclase activator $1 \mathrm{~A}$ gene-encoding guanylate cyclase activating protein-1. Arch Ophthalmol 2001;119:96-105.

17. Udar N, Yelchits S, Chalukya M, et al. Identification of GUCY2D gene mutations in CORD5 families and evidence of incomplete penetrance. Hum Mutat 2003;21:170-1.

18. Perrault I, Rozet JM, Gerber S, et al. A retGC-1 mutation in autosomal dominant conerod dystrophy. Am J Hum Genet 1998;63:651-4.

19. Payne AM, Morris AG, Downes SM, et al. Clustering and frequency of mutations in the retinal guanylate cyclase (GUCY2D) gene in patients with dominant cone-rod dystrophies. J Med Genet 2001;38:611-4.

20. Yang Z, Chen Y, Lillo C, et al. Mutant prominin 1 found in patients with macular degeneration disrupts photoreceptor disk morphogenesis in mice. J Clin Invest 2008; 118:2908-16.

21. Travis GH, Sutcliffe JG, Bok D. The retinal degeneration slow (rds) gene product is a photoreceptor disc membrane-associated glycoprotein. Neuron 1991;6:61-70.

22. Rattner A, Smallwood PM, Williams J, et al. A photoreceptor-specific cadherin is essential for the structural integrity of the outer segment and for photoreceptor survival. Neuron 2001;32:775-86.

23. Jensen VL, Carter $\mathrm{S}$, Sanders AA, et al. Whole-organism developmental expression profiling identifies rab-28 as a novel ciliary GTPase associated with the bbsome and intraflagellar transport. PLoS Genet 2016;12:e1006469.

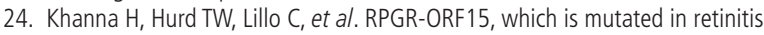
pigmentosa, associates with SMC1, SMC3, and microtubule transport proteins. J Biol Chem 2005;280:33580-7.
25. Higashide T, McLaren MJ, Inana G. Localization of HRG4, a photoreceptor protein homologous to Unc-119, in ribbon synapse. Invest Ophthalmol Vis Sci 1998;39:690-8.

26. Takao-Rikitsu E, Mochida S, Inoue E, et al. Physical and functional interaction of the active zone proteins, CAST, RIM1, and Bassoon, in neurotransmitter release. J Cell Biol 2004; 164:301-11.

27. Kellner U, Sadowski B, Zrenner E, et al. Selective cone dystrophy with protan genotype. Invest Ophthalmol Vis Sci 1995:36:2381-7.

28. Went LN, van Schooneveld MJ, Oosterhuis JA. Late onset dominant cone dystrophy with early blue cone involvement. J Med Genet 1992;29:295-8.

29. Thiadens AA, Phan TM, Zekveld-Vroon RC, et al. Clinical course, genetic etiology, and visual outcome in cone and cone-rod dystrophy. Ophthalmology 2012;119:819-26.

30. Langwińska-Wośko E, Szulborski K, Zaleska-Żmijewska A, et al. Electrophysiological testing as a method of cone-rod and cone dystrophy diagnoses and prediction of disease progression. Doc Ophthalmol 2015;130:103-9.

31. Wu H, Cowing JA, Michaelides M, et al. Mutations in the gene KCNV2 encoding a voltage-gated potassium channel subunit cause "cone dystrophy with supernormal rod electroretinogram" in humans. Am J Hum Genet 2006;79:574-9.

32. Stockman A, Henning GB, Michaelides M, et al. Cone dystrophy with "supernormal" rod ERG: psychophysical testing shows comparable rod and cone temporal sensitivity losses with no gain in rod function. Invest Ophthalmol Vis Sci 2014;55:832-40.

33. Krill AE, Deutman AF, Fishman M. The cone degenerations. Doc Ophthalmol 1973;35:1-80.

34. Uliss AE, Moore AT, Bird AC. The dark choroid in posterior retinal dystrophies. Ophthalmology 1987;94:1423-7

35. Heckenlively JR, Weleber RG. X-linked recessive cone dystrophy with tapetal-like sheen. A newly recognized entity with Mizuo-Nakamura phenomenon. Arch Ophthalmol 1986;104:1322-8.

36. Oishi M, Oishi A, Ogino K, et al. Wide-field fundus autofluorescence abnormalities and visual function in patients with cone and cone-rod dystrophies. Invest Ophthalmol Vis Sci 2014;55:3572-7.

37. Oishi A, Oishi M, Ogino K, et al. Wide-Field Fundus Autofluorescence for Retinitis Pigmentosa and Cone/Cone-Rod Dystrophy. Adv Exp Med Biol 2016;854:307-13.

38. Robson AG, Michaelides M, Saihan Z, et al. Functional characteristics of patients with retinal dystrophy that manifest abnormal parafoveal annuli of high density fundus autofluorescence; a review and update. Doc Ophthalmol 2008;116:79-89.

39. Lima LH, Sallum JM, Spaide RF. Outer retina analysis by optical coherence tomography in cone-rod dystrophy patients. Retina 2013;33:1877-80.

40. Inui E, Oishi A, Oishi M, et al. Tomographic comparison of cone-rod and rod-cone retinal dystrophies. Graefes Arch Clin Exp Ophthalmol 2014;252:1065-9.

41. Sergouniotis PI, Holder GE, Robson AG, et al. High-resolution optical coherence tomography imaging in KCNV2 retinopathy. Br J Ophthalmol 2012;96:213-7.

42. Georgiou M, Kalitzeos $A$, Patterson EJ, et al. Adaptive optics imaging of inherited retinal diseases. Br J Ophthalmol 2018;102:1028-35.

43. Wolfing Jl, Chung M, Carroll J, et al. High-resolution retinal imaging of cone-rod dystrophy. Ophthalmology 2006;113:1014-9.

44. Warren C, Scoles DH, Dubis A. Imaging cone structure in autosomal dominant cone rod dystrophy caused by GUCY2D m utations. Invest Ophth Vis Sci 2014;55:1102-02.

45. Langlo CS, Erker LR, Parker M, et al. Repeatability and longitudinal assessment of foveal cone structure in cngb3-associated achromatopsia. Retina 2017;37:1956-66.

46. Subbaraya I, Ruiz CC, Helekar BS, et al. Molecular characterization of human and mouse photoreceptor guanylate cyclase-activating protein (GCAP) and chromosomal localization of the human gene. J Biol Chem 1994;269:31080-9.

47. Palczewski K, Sokal I, Baehr W. Guanylate cyclase-activating proteins: structure, function, and diversity. Biochem Biophys Res Commun 2004;322:1123-30.

48. Wilkie SE, Li Y, Deery EC, et al. Identification and functional consequences of a new mutation (E155G) in the gene for GCAP1 that causes autosomal dominant cone dystrophy. Am J Hum Genet 2001;69:471-80.

49. Nong $E$, Lee W, Merriam JE, et al. Disease progression in autosomal dominant cone-rod dystrophy caused by a novel mutation (D100G) in the GUCA1A gene. Doc Ophthalmol 2014;128:59-67.

50. Kitiratschky VB, Behnen P, Kellner U, et al. Mutations in the GUCA1A gene involved in hereditary cone dystrophies impair calcium-mediated regulation of guanylate cyclase. Hum Mutat 2009;30:E782-E796.

51. Stockman A, Henning GB, Moore AT, et al. Visual consequences of molecular changes in the guanylate cyclase-activating protein. Invest Ophthalmol Vis Sci 2014;55:1930-40.

52. Chen $X$, Sheng $X$, Zhuang $W$, et al. GUCA1A mutation causes maculopathy in a fivegeneration family with a wide spectrum of severity. Genet Med 2017;19:945-54.

53. Kachi S, Nishizawa Y, Olshevskaya E, et al. Detailed localization of photoreceptor guanylate cyclase activating protein-1 and -2 in mammalian retinas using light and electron microscopy. Exp Eye Res 1999;68:465-73.

54. Song $H$, Rossi EA, Stone E, et al. Phenotypic diversity in autosomal-dominant cone-rod dystrophy elucidated by adaptive optics retinal imaging. Br J Ophthalmol 2018;102:136-41.

55. Ma J, Norton JC, Allen AC, et al. Retinal degeneration slow (rds) in mouse results from simple insertion of a thaplotype-specific element into protein-coding exon II. Genomics 1995;28:212-9. 
56. Loewen CJ, Molday RS. Disulfide-mediated oligomerization of Peripherin/Rds and Rom-1 in photoreceptor disk membranes. Implications for photoreceptor outer segment morphogenesis and degeneration. J Biol Chem 2000;275:5370-8.

57. Nakazawa M, Kikawa E, Chida Y, et al. Autosomal dominant cone-rod dystrophy associated with mutations in codon 244 (Asn244His) and codon 184 (Tyr184Ser) of the peripherin/RDS gene. Arch Ophthalmol 1996;114:72-8.

58. Nakazawa M, Naoi N, Wada Y, et al. Autosomal dominant cone-rod dystrophy associated with a Val200Glu mutation of the peripherin/RDS gene. Retina 1996;16:405-10.

59. Michaelides M, Holder GE, Bradshaw K, et al. Cone-rod dystrophy, intrafamilial variability, and incomplete penetrance associated with the R172W mutation in the peripherin/RDS gene. Ophthalmology 2005;112:1592-8.

60. Downes SM, Fitzke FW, Holder GE, et al. Clinical features of codon 172 RDS macular dystrophy: similar phenotype in 12 families. Arch Ophthalmol 1999;117:1373-83.

61. Gamundi MJ, Hernan I, Muntanyola M, et al. High prevalence of mutations in peripherin/RDS in autosomal dominant macular dystrophies in a Spanish population. Mol Vis 2007;13:1031-7.

62. Anand S, Sheridan E, Cassidy F, et al. Macular dystrophy associated with the Arg172Trp substitution in peripherin/RDS: genotype-phenotype correlation. Retina 2009;29:682-8.

63. Duncan JL, Talcott KE, Ratnam K, et al. Cone structure in retinal degeneration associated with mutations in the peripherin/RDS gene. Invest Ophthalmol Vis Sci 2011;52:1557-66.

64. Gocho K, Akeo K, Itoh N, et al. High-resolution adaptive optics retinal image analysis at early stage central areolar choroidal dystrophy with PRPH2 mutation. Ophthalmic Surg Lasers Imaging Retina 2016;47:1115-26.

65. Allikmets R, Singh N, Sun $H$, et al. A photoreceptor cell-specific ATP-binding transporter gene $(A B C R)$ is mutated in recessive Stargardt macular dystrophy. Nat Genet 1997; 15:236-46.

66. Tanna P, Strauss RW, Fujinami K, et al. Stargardt disease: clinical features, molecular genetics, animal models and therapeutic options. Br J Ophthalmol 2017;101:25-30.

67. Lambertus S, van Huet RAC, Bax NM, et al. Early-onset stargardt disease. Ophthalmology 2015;122:335-44.

68. Fujinami K, Zernant J, Chana RK, et al. Clinical and molecular characteristics of childhood-onset Stargardt disease. Ophthalmology 2015;122:326-34.

69. Fujinami K, Sergouniotis Pl, Davidson AE, et al. Clinical and molecular analysis of Stargardt disease with preserved foveal structure and function. Am J Ophthalmol 2013; 156:487-501.

70. van Huet RA, Bax NM, Westeneng-Van Haaften SC, et al. Foveal sparing in Stargardt disease. Invest Ophthalmol Vis Sci 2014;55:7467-78.

71. Fujinami K, Sergouniotis PI, Davidson AE, et al. The clinical effect of homozygous ABCA4 alleles in 18 patients. Ophthalmology 2013;120:2324-31.

72. Burke TR, Fishman GA, Zernant J, et al. Retinal phenotypes in patients homozygous for the G1961E mutation in the ABCA4 gene. Invest Ophthalmol Vis Sci 2012;53:4458-67.

73. Fujinami K, Lois N, Mukherjee R, et al. A longitudinal study of Stargardt disease: quantitative assessment of fundus autofluorescence, progression, and genotype correlations. Invest Ophthalmol Vis Sci 2013:54:8181-90.

74. Westeneng-van Haaften SC, Boon CJF, Cremers FPM, et al. Clinical and genetic characteristics of late-onset stargardt's disease. Ophthalmology 2012;119:1199-210.

75. Michaelides M, Chen LL, Brantley MA. ABCA4 mutations and discordant ABCA4 alleles in patients and siblings with bull's-eye maculopathy. Br J Ophthalmol 2007:91:1650-5

76. Strauss RW, Muñoz B, Wolfson Y, et al. Assessment of estimated retinal atrophy progression in stargardt macular dystrophy using spectral-domain optical coherence tomography. Br J Ophthalmol 2016;100:956-62.

77. Chen Y, Ratnam K, Sundquist SM, et al. Cone photoreceptor abnormalities correlate with vision loss in patients with Stargardt disease. Invest Ophthalmol Vis Sci 2011:52:3281-92.
78. Song $H$, Rossi EA, Latchney L, et al. Cone and rod loss in Stargardt disease revealed by adaptive optics scanning light ophthalmoscopy. JAMA Ophthalmol 2015;133:1198-203.

79. Ghosh AK, Murga-Zamalloa CA, Chan L, et al. Human retinopathy-associated ciliary protein retinitis pigmentosa GTPase regulator mediates cilia-dependent vertebrate development. Hum Mol Genet 2010;19:90-8.

80. Vervoort R, Lennon A, Bird AC, et al. Mutational hot spot within a new RPGR exon in X-linked retinitis pigmentosa. Nat Genet 2000;25:462-6.

81. Shu X, Black GC, Rice JM, et al. RPGR mutation analysis and disease: an update. Hum Mutat 2007:28:322-8.

82. Ebenezer ND, Michaelides M, Jenkins SA, et al. Identification of novel RPGR ORF15 mutations in X-linked progressive cone-rod dystrophy (XLCORD) families. Invest Ophthalmol Vis Sci 2005;46:1891-8.

83. Tee JJ, Smith AJ, Hardcastle AJ, et al. RPGR-associated retinopathy: clinical features, molecular genetics, animal models and therapeutic options. Br J Ophthalmol 2016;100:1022-7.

84. Yang $L$, Yin $X$, Feng $L$, et al. Novel mutations of RPGR in Chinese retinitis pigmentosa patients and the genotype-phenotype correlation. PLoS One 2014;9:e85752.

85. Thiadens AA, Soerjoesing GG, Florijn RJ, et al. Clinical course of cone dystrophy caused by mutations in the RPGR gene. Graefes Arch Clin Exp Ophthalmol 2011;249:1527-35.

86. Talib M, van Schooneveld MJ, Thiadens AA, et al. Clinical and genetic characteristics of male patients with rpgr-associated retinal dystrophies. Retina 2018:1.

87. Robson AG, Michaelides M, Luong VA, et al. Functional correlates of fundus autofluorescence abnormalities in patients with RPGR or RIMS1 mutations causing cone or cone rod dystrophy. Br J Ophthalmol 2008;92:95-102.

88. JJL T, Kalitzeos A, Webster AR. Quantitative analysis of hyperautofluorescent rings to characterize the natural history and progression in rpgr-associated retinopathy. Retina 2017.

89. Smith J, Ward D, Michaelides M, et al. New and emerging technologies for the treatment of inherited retinal diseases: a horizon scanning review. Eye 2015;29:1131-40.

90. Mata NL, Weng J, Travis GH. Biosynthesis of a major lipofuscin fluorophore in mice and humans with ABCR-mediated retinal and macular degeneration. Proc Natl Acad SciU SA 2000:97:7154-9.

91. Sparrow JR, Zhou J, Cai B. DNA is a target of the photodynamic effects elicited in A2E laden RPE by blue-light illumination. Invest Ophthalmol Vis Sci 2003;44:2245-51.

92. Sparrow JR, Vollmer-Snarr HR, Zhou J, et al. A2E-epoxides damage DNA in retinal pigment epithelial cells. Vitamin $E$ and other antioxidants inhibit A2E-epoxide formation. J Biol Chem 2003;278:18207-13.

93. Jiang $L$, Zhang $H$, Dizhoor $A M$, et al. Long-term RNA interference gene therapy in a dominant retinitis pigmentosa mouse model. Proc Natl Acad Sci U SA 2011;108:18476-81.

94. Sarra GM, Stephens C, de Alwis M, et al. Gene replacement therapy in the retinal degeneration slow (rds) mouse: the effect on retinal degeneration following partial transduction of the retina. Hum Mol Genet 2001;10:2353-61.

95. Han Z, Conley SM, Makkia RS, et al. DNA nanoparticle-mediated ABCA4 delivery rescues Stargardt dystrophy in mice. J Clin Invest 2012;122:3221-6.

96. Beltran WA, Cideciyan AV, Lewin AS, et al. Gene augmentation for X-linked retinitis pigmentosa caused by mutations in RPGR. Cold Spring Harb Perspect Med 2014;5:a017392.

97. Kumaran N, Michaelides M, Smith AJ, et al. Retinal gene therapy. Br Med Bull 2018;126:13-25.

98. Bryant L, Lozynska O, Maguire AM, et al. Prescreening whole exome sequencing results from patients with retinal degeneration for variants in genes associated with retinal degeneration. Clin Ophthalmol 2018;12:49-63.

99. Carss KJ, Arno G, Erwood M, et al. Comprehensive Rare Variant Analysis via WholeGenome Sequencing to Determine the Molecular Pathology of Inherited Retinal Disease. Am J Hum Genet 2017:100:75-90. 